\title{
A convenient route to 3 -substituted isothiazoles using nitrile sulfide cycloaddition chemistry
}

\author{
John Crosby, Marion C. McKie, R. Michael Paton, ${ }^{*}$ and John F. Ross \\ Department of Chemistry, The University of Edinburgh, West Mains Road, Edinburgh, \\ EH9 3JJ, UK \\ E-mail: R.M.Paton@ed.ac.uk
}

\begin{abstract}
Dedicated to Otto Meth-Cohn on the Occasion of his $65^{\text {th }}$ birthday (received 15 Jun 00; accepted 03 Oct 00; published on the web 11 Oct 00)
\end{abstract}

\begin{abstract}
Isothiazole-3- and 4-carboxylate esters are readily prepared by 1,3-dipolar cycloaddition of nitrile sulfides, generated by thermal decarboxylation of the corresponding 1,3,4oxathiazol-2-ones, to acrylate, fumarate and maleate esters, followed by phase-transfermediated hypochlorite oxidation of the resulting 2-isothiazoline cycloadducts.
\end{abstract}

Keywords: Isothiazoles, nitrile sulfide, 1,3-dipolar cycloaddition, hypochlorite oxidation.

\section{Introduction}

Isothiazoles show a wide spectrum of biological activity ${ }^{1}$ and there is therefore interest in effective methods for their synthesis. One route that has been used ${ }^{2,3}$ with some success involves the 1,3-dipolar cycloaddition of nitrile sulfides $1^{4}$ to alkynes. Nitrile sulfides can be generated by thermal decarboxylation of the corresponding 1,3,4oxathiazol-2-one 2, which are readily prepared from carboxamides (Scheme 1). The range of suitable alkynes, however, is limited by low reactivity and, in some cases, by thermal instability. We have investigated an alternative nitrile sulfide-based approach involving initial cycloaddition to alkenes, which are generally more accessible and of greater reactivity, followed by oxidation of the resulting 2-isothiazoline cycloadducts (Scheme 1). 


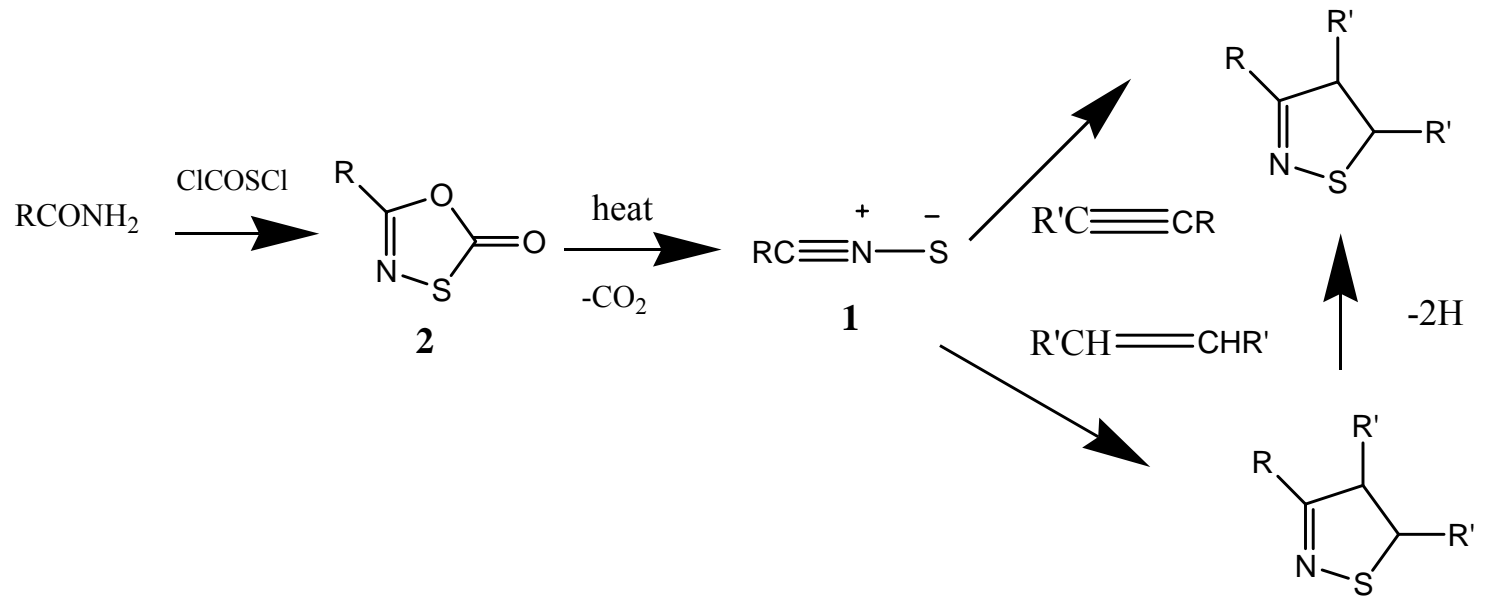

\section{Scheme 1}

\section{Results and Discussion}

Cycloaddition of Nitrile Sulfides to Alkenes.

Nitrile sulfides are short-lived species prone to fragmentation to sulfur and the corresponding nitrile, and it is therefore necessary for them to be generated in situ in the presence of the dipolarophile. ${ }^{4}$ In a typical experiment a solution of phenyloxathiazolone $2 \mathrm{a}$ in xylene was heated under reflux $\left(\sim 138{ }^{\circ} \mathrm{C}\right)$ with ten equivalents of diethyl fumarate until HPLC analysis showed that all the starting material had been consumed $(4.5 \mathrm{~h})$. Removal of the solvent and excess dipolarophile afforded an oil from which 2 -isothiazoline 3 a $(70 \%)$ was isolated by crystallisation. The trans arrangement of the ethoxycarbonyl groups in the product was evident from the $4 \mathrm{~Hz}$ coupling between 4-H and 5-H in the ${ }^{1} \mathrm{H}$ NMR spectrum, which is comparable to those reported for the dimethyl fumarate adduct $4 \mathrm{a}^{5}$ and the corresponding 2-isoxazoline derived from reaction of diethyl fumarate with benzonitrile oxide $\left(\mathrm{PhC}^{\circ} \mathrm{N}^{+}-\mathrm{O}^{-}\right){ }^{6}{ }^{6}$ The structure of adduct $3 \mathrm{a}$ was confirmed by X-ray crystallography. ${ }^{7}$ 4-Methoxy-, 4-methyl, and 4-chloro-benzonitrile sulfides $1 \mathrm{~b}-\mathrm{d}$, and alkanonitrile sulfides $1 \mathrm{e}-\mathrm{g}$ reacted similarly (Table 1, entries 2-7). A noteworthy feature of these results is the differing times required for the reaction to go to completion; electron-withdrawing substituents decrease the rate of reaction, while electron-donation has the opposite effect in the order $\mathrm{Me}>\mathrm{Pr}>$ heptyl $>4-\mathrm{MeOC}_{6} \mathrm{H}_{4}>4-\mathrm{MeC}_{6} \mathrm{H}_{4}>\mathrm{Ph}>>4-\mathrm{ClC}_{6} \mathrm{H}_{4}$. This effect can be attributed $^{2 a}$ to the development of a partial positive charge at the 5-position of the oxathiazolone ring in the transition state for the decarboxylation 


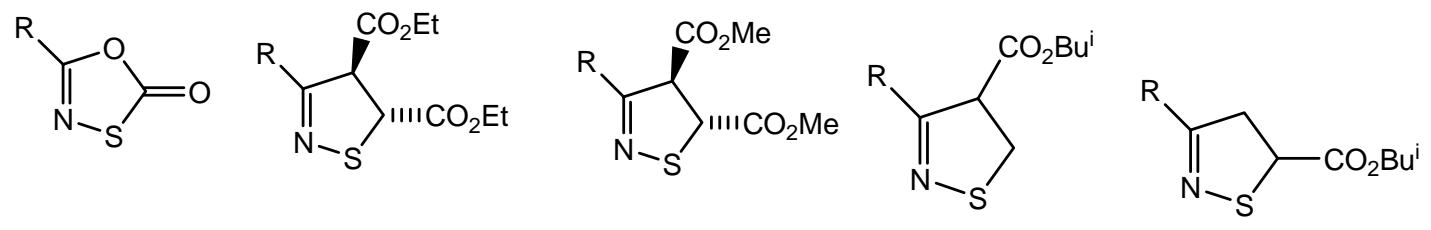

$\left[1-4,7,8: \mathrm{R}=\mathrm{a}, \mathrm{Ph} ; \mathrm{b}, 4-\mathrm{MeOC}_{6} \mathrm{H}_{4} ; \mathrm{c}, 4-\mathrm{MeC}_{6} \mathrm{H}_{4} ; \mathrm{d}, 4-\mathrm{ClC}_{6} \mathrm{H}_{4}\right.$; e, Me; f, Pr; g, $\left.\mathrm{CH}_{3}\left(\mathrm{CH}_{2}\right)_{6}\right]$

Table 1.Cycloaddition reactions of nitrile sulfides $\left(\mathrm{RC}^{\circ} \mathrm{N}^{+}-\mathrm{S}^{-}\right)$to alkenes at $135-140{ }^{\circ} \mathrm{C}$

\begin{tabular}{|c|c|c|c|c|c|c|c|c|}
\hline \multirow{2}{*}{ Entry } & \multirow{2}{*}{$\mathrm{R}$} & \multirow{2}{*}{ alkene $^{a}$} & \multirow{2}{*}{$\begin{array}{c}\text { reactant } \\
\text { ratio }\end{array}$} & \multirow{2}{*}{$\begin{array}{c}\text { reaction } \\
\text { time/h }\end{array}$} & \multicolumn{3}{|c|}{ 2-isothiazoline(s) } & \multirow{2}{*}{$\begin{array}{c}\text { nitrile } \\
\text { yield/\% } \%\end{array}$} \\
\hline & & & & & & yield $/ \%$ & ratio $^{b, c}$ & \\
\hline 1 & $\mathrm{C}_{6} \mathrm{H}_{5}$ & DEF & $1: 10$ & 4.5 & $3 a$ & 70 & - & $\mathrm{d}$ \\
\hline 2 & $4-\mathrm{MeOC}_{6} \mathrm{H}_{4}$ & DEF & $1: 10$ & 2.5 & $3 b$ & 80 & - & $\mathrm{d}$ \\
\hline 3 & $4-\mathrm{MeC}_{6} \mathrm{H}_{4}$ & DEF & $1: 10$ & 4 & $3 c$ & 40 & - & $\mathrm{d}$ \\
\hline 4 & $4-\mathrm{ClC}_{6} \mathrm{H}_{4}$ & DEF & $1: 10$ & 9.5 & $3 d$ & 67 & - & $\mathrm{d}$ \\
\hline 5 & $\mathrm{Me}$ & $\mathrm{DEF}$ & $1: 10$ & 1.0 & $3 e$ & 68 & - & d \\
\hline 6 & $\mathrm{CH}_{3}\left(\mathrm{CH}_{2}\right)_{2}$ & DEF & $1: 10$ & 1.25 & $3 f$ & 69 & - & $\mathrm{d}$ \\
\hline 7 & $\mathrm{CH}_{3}\left(\mathrm{CH}_{2}\right)_{6}$ & DEF & $1: 10$ & 1.5 & $3 g$ & 70 & - & $\mathrm{d}$ \\
\hline 8 & $\mathrm{C}_{6} \mathrm{H}_{5}$ & DMF & $1: 4$ & 12 & $4 b$ & 61 & - & d \\
\hline 9 & $4-\mathrm{MeOC}_{6} \mathrm{H}_{4}$ & DEM & $1: 10$ & 5 & $3 b$ & $23,32^{c}$ & - & 67 \\
\hline 10 & $4-\mathrm{MeC}_{6} \mathrm{H}_{4}$ & DEM & $1: 8$ & 14 & $3 c$ & $<5^{c}$ & - & $>80^{c}$ \\
\hline 11 & $4-\mathrm{MeOC}_{6} \mathrm{H}_{4}$ & IBA & $1: 2$ & 5 & $7 b / 8 b$ & $36^{c}$ & 0.33 & 58 \\
\hline 12 & $4-\mathrm{MeOC}_{6} \mathrm{H}_{4}$ & IBA & $1: 3$ & 5 & $7 b / 8 b$ & $40^{c}$ & 0.35 & 53 \\
\hline 13 & $4-\mathrm{MeOC}_{6} \mathrm{H}_{4}$ & IBA & $1: 5$ & 5 & $7 b / 8 b$ & $45^{c}$ & 0.36 & 50 \\
\hline 14 & $4-\mathrm{MeOC}_{6} \mathrm{H}_{4}$ & IBA & $1: 6$ & 5 & $7 b / 8 b$ & $48^{c}$ & 0.36 & 46 \\
\hline 15 & $4-\mathrm{MeOC}_{6} \mathrm{H}_{4}$ & IBA & $1: 7$ & 5 & $7 b / 8 b$ & $51^{c}$ & 0.38 & 43 \\
\hline 16 & $4-\mathrm{MeOC}_{6} \mathrm{H}_{4}$ & IBA & $1: 9$ & 5 & $7 b / 8 b$ & $55^{c}$ & 0.37 & 40 \\
\hline 17 & $4-\mathrm{MeOC}_{6} \mathrm{H}_{4}$ & IBA & $1: 11$ & 5 & $7 b / 8 b$ & $59^{c}$ & 0.37 & 37 \\
\hline
\end{tabular}

${ }^{a}$ alkenes: (DEF) diethyl fumarate, (DMF) dimethyl fumarate, (DEM) diethyl maleate, (IBA) isobutyl acrylate

${ }^{b}$ 4-carboxylate (7b) : 5-carboxylate (8b)

${ }^{c}$ determined by HPLC analysis

${ }^{d}$ not determined

Having established that diethyl fumarate (DEF) is an efficient dipolarophile for trapping a range of nitrile sulfides, the corresponding reaction with diethyl maleate (DEM) was 
examined. 4-Methoxy-phenyloxathiazolone 2b and excess DEM (1:10) in xylene were heated under reflux for 5 hours. The only products detected (HPLC) and isolated were the trans-isothiazoline $3 \mathrm{~b}(32 \%)$ and 4-methoxybenzonitrile $(67 \%)$. The cycloadduct was identified from its $\mathrm{mp}$ (and mixed $\mathrm{mp}$ ) and its spectroscopic properties by comparison with those of the authentic compound prepared from DEF. Formation of nitriles as by-products is a common feature of nitrile sulfide reactions, particularly with less reactive dipolarophiles, and is attributed to fragmentation of the nitrile sulfide competing with cycloaddition. ${ }^{4}$ Similar results were obtained for the reaction of DEM with nitrile sulfide 1c. The low adduct yields are consistent with the reported ${ }^{8}$ lower reactivity of cis-alkenes. Two possibilities are considered for the formation of the trans adduct (Scheme 2): either the nitrile sulfide reacts with the cis-alkene and the resulting cis adduct rearranges to the thermodynamically more stable trans product, possibly via enolisation of the 4-carboxy group (path a); or the dipolarophile undergoes cis to trans isomerisation under the reaction conditions prior to cycloaddition (path b). There is precedent for the former explanation in the work of Rahman and Clapp ${ }^{6}$ who observed that, in the corresponding reaction of benzonitrile oxide with DEM, the expected cis adduct formed at room temperature rapidly rearranged to the trans isomer at $80{ }^{\circ} \mathrm{C}$. On the other hand, examination of the unreacted dipolarophile recovered from the reaction mixture by ${ }^{1} \mathrm{H}$ NMR spectroscopy showed the presence of DEF $\left(\delta_{=\mathrm{CH}} 6.4 \mathrm{ppm}, \mathrm{cf} 6.0\right.$ ppm for DEM), consistent with path (b). As DEM is thermally stable at the reaction temperature it is assumed that it results, either (i) from a reversible cycloaddition which allows the more stable trans-alkene to be generated in the reverse step, similar to that proposed $^{9}$ to explain DEF formation in the reaction of arylchlorocarbenes with DEM, or (ii) via an isomerisation process induced by a reactive sulfur species formed as a byproduct on decomposition of the nitrile sulfide.
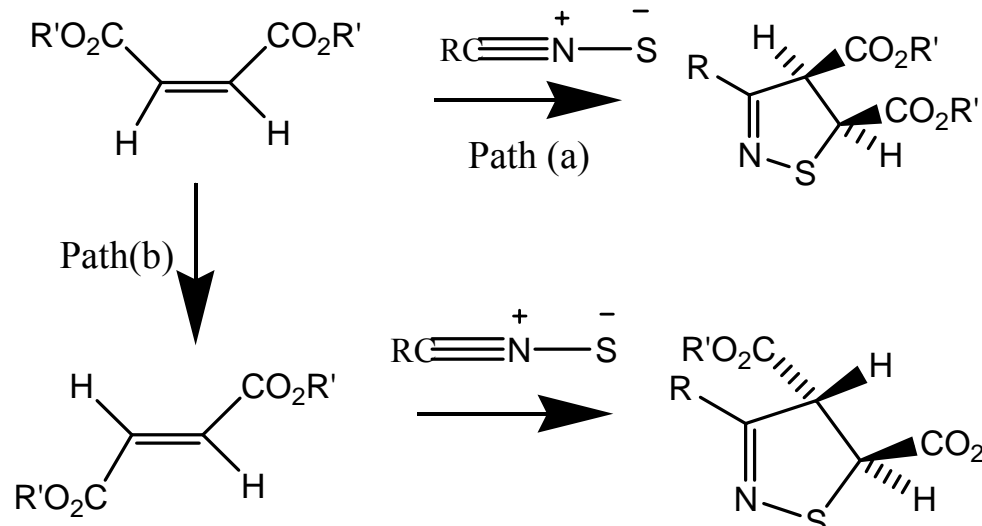

Scheme 2
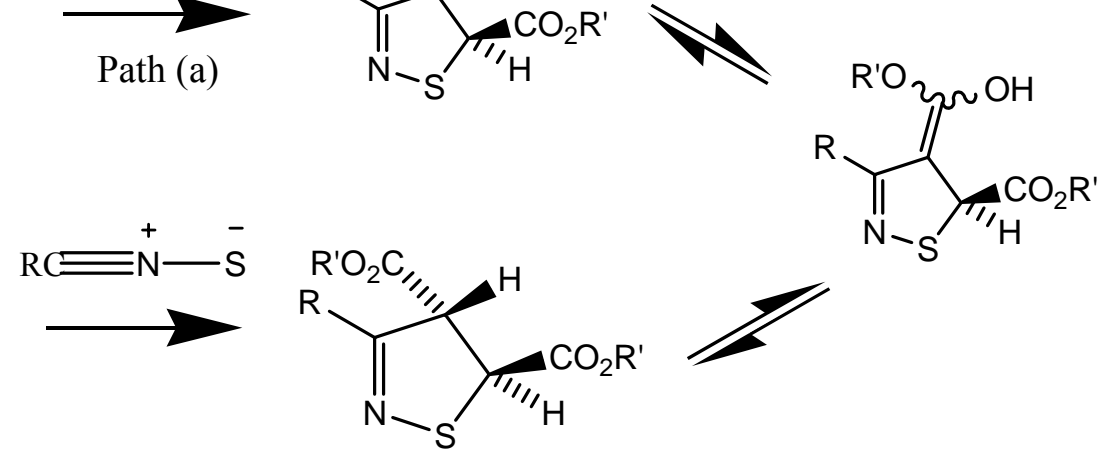

\section{Scheme 2}


Cis-trans dipolarophile isomerisation was also observed during the reaction of nitrile sulfide 2c with cis-1,2-bis(phenylsulfonyl)ethene (cis-PSE). Thermolysis of oxathiazolone $2 \mathrm{c}$ and cis-PSE (1:2.5) in refluxing xylene for $20 \mathrm{~h}$ afforded the 4(phenylsulfonyl)isothiazole 5 (38\%), rather than the expected isothiazoline 6 (Scheme 3). Presumably the initially formed isothiazoline undergoes spontaneous elimination of phenylsulfonic acid under the reaction conditions. 4-Methylbenzonitrile and sulfur were formed as by-products. The regiochemistry of the adduct was established from its ${ }^{1} \mathrm{H}$ NMR spectrum, which showed a signal at $9.5 \mathrm{ppm}$ characteristic for $5-\mathrm{H}$ of isothiazoles. The excess dipolarophile was recovered and shown from its NMR spectra and mp to have undergone complete conversion to the trans isomer (trans-PSE). cis-PSE was shown to be thermally stable at the reaction temperature $\left(135^{\circ} \mathrm{C}\right)$.
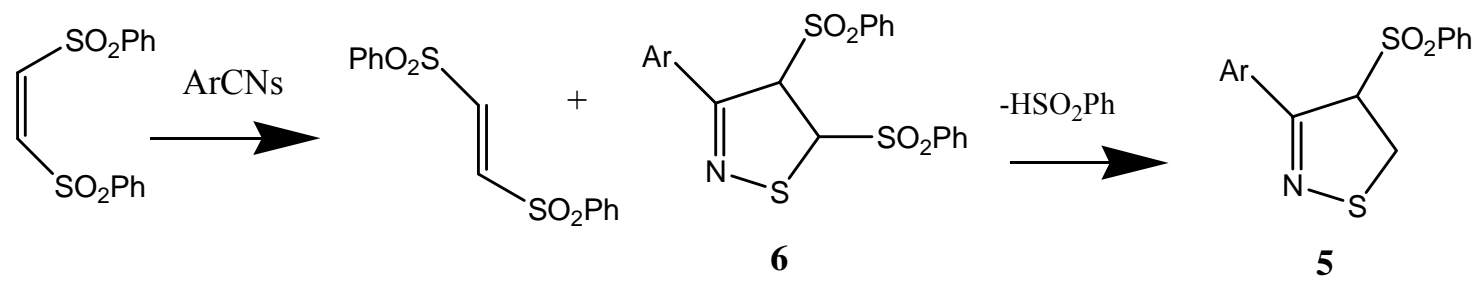

Scheme 3. $\left(\mathrm{Ar}=4-\mathrm{MeC}_{6} \mathrm{H}_{4}\right)$.

In order to help distinguish between the two isomerisation pathways (i,ii) a pair of olefinic dipolarophiles (cis-, trans-stilbene) were selected which were expected to be too unreactive to undergo cycloaddition with nitrile sulfides. Thermolysis of oxathiazolone $2 \mathrm{c}$ in the presence of a two-fold excess of trans-stilbene gave only sulfur (95\%) and 4-methylbenzonitrile (92\%) together with recovered trans-stilbene (90\%); no adduct could be detected by mass spectrometry. However, when cis-stilbene was used under similar conditions, sulfur (94\%) and 4-methylbenzonitrile $(90 \%)$ were isolated together with trans-stilbene (95\%). These results are consistent with pathway (ii) involving oxathiazolone decomposition product(s), presumably sulfur, inducing the cis to trans rearrangement. This hypothesis is supported by the observation that heating DEM with sulfur at $\sim 220^{\circ} \mathrm{C}$ resulted in complete conversion to DEF. The nature of the active species is uncertain, but may be reactive form of sulfur, eg $\mathrm{S}_{2}$ or $\mathrm{S}_{6}$, resulting from the decomposition of the nitrile sulfide. ${ }^{10}$

The reaction of 4-methoxybenzonitrile sulfide $1 \mathrm{~b}$ with isobutyl acrylate afforded 4methoxybenzonitrile and a regioisomeric mixture of isothiazoline-4- and 5-carboxylate 
esters $7 \mathrm{~b}$ and $8 \mathrm{~b}$. The cycloadduct to nitrile product ratio was found to be sensitive to the excess of dipolarophile used, varying form 0.62 for dipolarophile:dipole $=2: 1$ to 1.60 at 11:1 (Table 1, entries 10-17). The regioisomer ratio ( 0.35) remained constant within experimental error. The individual regioisomers were readily identified from their NMR spectra; eg the 4-carboxylate $7 \mathrm{~b}$ showed characteristic ${ }^{13} \mathrm{C}$ peaks for $\mathrm{CH}-4$ and $\mathrm{CH}_{2}-5$ at 56.9 and 37.4 ppm respectively, whereas order was reversed for the 5carboxylate isomer $8 \mathrm{~b}\left[\delta_{\mathrm{C}} 41.0\left(\mathrm{CH}_{2}-4\right), 48.8(\mathrm{CH}-5)\right]$. The modest regioselectivity observed for these nitrile sulfide / acrylate ester cycloadditions (25:75) differs markedly from that reported ${ }^{8}$ for the corresponding reactions of nitrile oxides, which are much more selective, typically giving isomer ratios of ca 5:95. The lower selectivity in the nitrile sulfide case can be attributed to greater dipole-HOMO control in the cycloaddition. $^{2 \mathrm{c}}$

\section{Oxidation of 2-isothiazolines to isothiazoles}

Previous work by Howe and Franz ${ }^{5}$ showed that isothiazoline $4 \mathrm{a}$ could be dehydrogenated to isothiazole $9 \mathrm{a}$ in moderate yield (40\%) using excess DDQ in refluxing chlorobenzene $\left(5 \mathrm{~h}\right.$ at $\left.\sim 130{ }^{\circ} \mathrm{C}\right)$. In order to avoid these forcing conditions and facilitate isolation of the product, we have explored the feasibility of using aqueous sodium hypochlorite in a two-phase reaction. In a pilot experiment a solution of isothiazoline $4 \mathrm{a}$ in dichloromethane was stirred vigorously with $8 \%$ aq. $\mathrm{NaOCl}$ at room temperature. TLC and HPLC analysis showed that after 3 weeks $4 \mathrm{a}$ had been transformed into $9 \mathrm{a}$, which was isolated in $92 \%$ yield (99\% by HPLC). Isothiazoline $3 \mathrm{~b}$ was converted to isothiazole $10 \mathrm{~b}(93 \%)$ similarly (Table 2 , entries $1 \& 2$ ).

Although these results showed that hypochlorite is an effective oxidising agent, the reaction times were unacceptably long. In order to accelerate the process benzyltriethylammonium chloride was added as a phase transfer catalyst. By this means the reaction times were greatly reduced; eg isothiazoline 3 a was converted to isothiazole 10 a within 5 hours and in high yield (92\%) (Table 2, entry 3). To illustrate the general utility of the method a series of oxidations were carried out using selected 3-aryl- and 3alkyl-isothiazoledicarboxylates, and isothiazole-4-, and -5-carboxylates $7 \mathrm{~b}$ and $8 \mathrm{~b}$ (entries 4-9). In all cases the reactions were complete in 5 hours or less, and the yields were good $(>80 \%)$. Attempted dehydrogenation of isothiazoline $4 \mathrm{a}$ using oxygen at room temperature, by thermolysis $\left(164{ }^{\circ} \mathrm{C}\right)$, and thermolysis in the presence of air or sulfur gave low conversions to isothiazole 9a (entries 10-13). However, when a combination of air or oxygen with sulfur was used moderate yields $(20 \%, 44 \%)$ of the isothiazole were achieved (entries 14,15). 


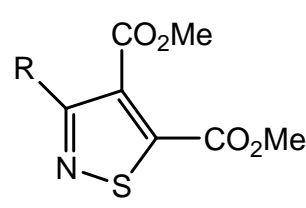

9

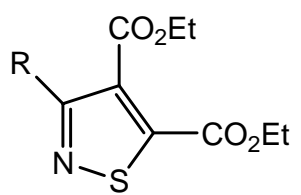

10

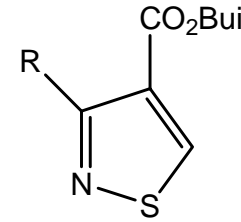

11

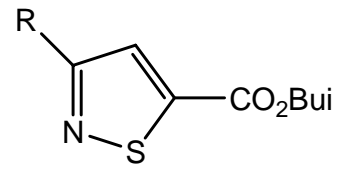

12

[9-12: $\mathrm{R}=\mathrm{a}, \mathrm{Ph} ; \mathrm{b}, 4-\mathrm{MeOC}_{6} \mathrm{H}_{4} ; \mathrm{c}, 4-\mathrm{MeC}_{6} \mathrm{H}_{4} ; \mathrm{d}, 4-\mathrm{ClC}_{6} \mathrm{H}_{4} ; \mathrm{e}, \mathrm{Me} ; \mathrm{f}, \mathrm{Pr} ; \mathrm{g}, \mathrm{CH}_{3}\left(\mathrm{CH}_{2}\right)_{6}$ ]

Table 2. Oxidation of 2-isothiazolines to isothiazoles

\begin{tabular}{cccccccc}
\hline \multirow{2}{*}{ Entry } & \multirow{2}{*}{ 2-isothiazoline } & \multicolumn{2}{c}{ reaction conditions } & \multicolumn{2}{c}{ isothiazole } & unreacted \\
\cline { 3 - 6 } & & reagent $^{a}$ & time & temp. $/{ }^{\circ} \mathrm{C}$ & & yield $/ \%$ & isothiazoline \\
\hline 1 & $4 \mathrm{a}$ & $\mathrm{NaOCl}$ & $21 \mathrm{~d}$ & $\sim 18$ & $9 \mathrm{a}$ & $92,99^{c}$ & $b$ \\
2 & $3 \mathrm{~b}$ & $\mathrm{NaOCl}$ & $21 \mathrm{~d}$ & $\sim 18$ & $10 \mathrm{~b}$ & 93 & $b$ \\
3 & $3 \mathrm{a}$ & $\mathrm{NaOCl}+$ cat. & $5 \mathrm{~h}$ & $\sim 18$ & $10 \mathrm{a}$ & 92 & $b$ \\
4 & $3 \mathrm{~d}$ & $\mathrm{NaOCl}+$ cat. & $5 \mathrm{~h}$ & $\sim 18$ & $10 \mathrm{~d}$ & 94 & $b$ \\
5 & $3 \mathrm{e}$ & $\mathrm{NaOCl}+$ cat. & $5 \mathrm{~h}$ & $\sim 18$ & $10 \mathrm{e}$ & 85 & $b$ \\
6 & $3 \mathrm{f}$ & $\mathrm{NaOCl}+$ cat. & $5 \mathrm{~h}$ & $\sim 18$ & $10 \mathrm{f}$ & 81 & $b$ \\
7 & $3 \mathrm{~g}$ & $\mathrm{NaOCl}+$ cat. & $5 \mathrm{~h}$ & $\sim 18$ & $10 \mathrm{~g}$ & 80 & $b$ \\
8 & $7 \mathrm{~b}$ & $\mathrm{NaOCl}+$ cat. & $4.5 \mathrm{~h}$ & $\sim 18$ & $11 \mathrm{~b}$ & 80 & $b$ \\
9 & $8 \mathrm{~b}$ & $\mathrm{NaOCl}+$ cat. & $4 \mathrm{~h}$ & $\sim 18$ & $12 \mathrm{~b}$ & 86 & $b$ \\
10 & $4 \mathrm{a}$ & $\mathrm{O}_{2}$ & $26 \mathrm{~h}$ & $\sim 18$ & $9 \mathrm{a}$ & $<1$ & 100 \\
11 & $4 \mathrm{a}$ & $\mathrm{N}_{2}$ & $24 \mathrm{~h}$ & $164^{d}$ & $9 \mathrm{a}$ & $2^{c}$ & $98^{c}$ \\
12 & $4 \mathrm{a}$ & $\mathrm{air}$ & $24 \mathrm{~h}$ & $164^{d}$ & $9 \mathrm{a}$ & $4^{c}$ & $95^{c}$ \\
13 & $4 \mathrm{a}$ & $\mathrm{N}_{2}+\mathrm{S}_{8}$ & $24 \mathrm{~h}$ & $164^{d}$ & $9 \mathrm{a}$ & $3^{c}$ & $97^{c}$ \\
14 & $4 \mathrm{a}$ & $\mathrm{air}+\mathrm{S}_{8}$ & $48 \mathrm{~h}$ & $164^{d}$ & $9 \mathrm{a}$ & $20^{c}$ & $79^{c}$ \\
15 & $4 \mathrm{a}$ & $\mathrm{O}_{2}+\mathrm{S}_{8}$ & $48 \mathrm{~h}$ & $164^{d}$ & $9 \mathrm{a}$ & $44^{c}$ & $52^{c}$ \\
\hline
\end{tabular}

${ }^{a} 8 \%$ aq. $\mathrm{NaOCl}$, cat. $\mathrm{BnN}^{+} \mathrm{Et}_{3} \cdot \mathrm{Cl}^{-}$

${ }^{b}$ not detected

${ }^{c}$ determined by HPLC analysis

${ }^{d}$ in mesitylene under reflux

In conclusion, for ease of work-up and high product yields phase-transfer mediated hypochlorite oxidation at room-temperature of 2-isothiazolines to isothiazoles is more effective than dehydrogenation with DDQ. The two-step route to isothiazoles involving 
nitrile sulfide cycloaddition to electron-deficient alkenes, followed by oxidation of the resulting isothiazoline cycloadduct thus affords isothiazoles in $55-80 \%$ overall yield.

\section{Experimental Section}

General Procedures. The instrumentation for recording IR, ${ }^{1} \mathrm{H}$ and ${ }^{13} \mathrm{C} N M R$, and mass spectra, and the analytical methods used for monitoring the reactions were as previously described. ${ }^{11,12}$ Oxathiazolones $2 \mathrm{a}-2 \mathrm{f}^{3}$ and dimethyl 3-phenylisothiazole-4,5dicarboxylate $(9 a)^{2 a}$ were prepared as previously reported. NMR spectra were recorded in $\mathrm{CDCl}_{3}$ unless otherwise stated.

5-Heptyl-1,3,4-oxathiazol-2-one (2g). This compound was prepared (61\%) from octanamide and chlorocarbonylsulfenyl chloride as previously described ${ }^{3}$ for the propyl analogue 2f. Bp $94{ }^{\circ} \mathrm{C}$ at $1.0 \mathrm{mmHg}$ (Found: $\mathrm{C}, 53.9 ; \mathrm{H}, 7.7 ; \mathrm{N}, 7.1 . \mathrm{C}_{9} \mathrm{H}_{15} \mathrm{NO}_{2} \mathrm{~S}$ requires $\mathrm{C}, 53.7 ; \mathrm{H}, 7.5 ; \mathrm{N}, 7.0 \%) ; v_{\max }\left(\right.$ film) $1765 \mathrm{~cm}^{-1}(\mathrm{C}=\mathrm{O})$.

Reaction of nitrile sulfides with dialkyl fumarates. The general procedure was to heat under reflux $\left(\sim 138^{\circ} \mathrm{C}\right)$ a solution of the 1,3,4-oxathiazol-2-one (2) (24 mmol) and the alkene $(240 \mathrm{mmol})$ in dry xylene. The reaction was continued until HPLC analysis showed that all the starting material had been consumed. After evaporation under reduced pressure to remove the solvent and excess dipolarophile, the dialkyl (E)-2isothiazoline-4,5-dicarboxylatewas separated from nitrile and sulfur by-products by distillation and/or recrystallisation. Reaction conditions and product yields are given in Table 1.

Diethyl (E)-3-phenyl-2-isothiazoline-4,5-dicarboxylate (3a). Mp $62-63{ }^{\circ} \mathrm{C}$ (from EtOH) (Found: C, 58.4; H, 5.6; N, 4.5. $\mathrm{C}_{15} \mathrm{H}_{17} \mathrm{NO}_{4} \mathrm{~S}$ requires $\mathrm{C}, 58.6$; $\mathrm{H}, 5.6$; N, 4.6\%); $v_{\max }$ (Nujol) $1732 \mathrm{~cm}^{-1}(\mathrm{C}=\mathrm{O}) ; \delta_{\mathrm{H}}$ 7.88-7.56 (m, 2H, $\left.\mathrm{PhH}\right), 7.42-7.24(\mathrm{~m}, 3 \mathrm{H}, \mathrm{PhH})$, $5.17(\mathrm{~d}, 1 \mathrm{H}, J=4 \mathrm{~Hz}, 5-\mathrm{H}), 4.76(\mathrm{~d}, 1 \mathrm{H}, J=4 \mathrm{~Hz}, 4-\mathrm{H}), 4.17\left(\mathrm{q}, 2 \mathrm{H}, \mathrm{OCH}_{2}\right), 4.10$ (q, $\left.2 \mathrm{H}, \mathrm{OCH}_{2}\right), 1.24\left(\mathrm{t}, 3 \mathrm{H}, \mathrm{CH}_{3}\right), 1.07\left(\mathrm{t}, 3 \mathrm{H}, \mathrm{CH}_{3}\right) ; \delta_{\mathrm{C}} 170.4,168.5(\mathrm{C}=\mathrm{O}), 161.7(\mathrm{C}-3)$, $133.1(\mathrm{PhC}), 130.2,128.4,127.9(5 \mathrm{PhCH}), 62.3,62.1\left(\mathrm{OCH}_{2}\right), 59.3(\mathrm{C}-5), 53.6(\mathrm{C}-4)$, 14.0, $13.8\left(\mathrm{CH}_{3}\right) ; \mathrm{m} / \mathrm{z} 307\left(M^{+}\right), 188,135\left(\mathrm{PhCNS}^{+}\right), 103\left(\mathrm{PhCN}^{+}\right)$.

Diethyl (E)-3-(4-methoxyphenyl)-2-isothiazoline-4,5-dicarboxylate (3b). Mp 45$46{ }^{\circ} \mathrm{C}$ (from EtOH) (Found: $\mathrm{C}, 57.1 ; \mathrm{H}, 5.7 ; \mathrm{N}, 4.0 . \mathrm{C}_{16} \mathrm{H}_{19} \mathrm{NO}_{5} \mathrm{~S}$ requires $\mathrm{C}, 57.0 ; \mathrm{H}$, 5.8; N, 4.1\%); $v_{\max }$ (Nujol) $1731 \mathrm{~cm}^{-1}(\mathrm{C}=\mathrm{O}) ; \delta_{\mathrm{H}} 7.70(\mathrm{~d}, 2 \mathrm{H}, J=9 \mathrm{~Hz}, \mathrm{ArH}), 6.83(\mathrm{~d}$, $2 \mathrm{H}, J=9 \mathrm{~Hz}, \mathrm{ArH}), 5.08(\mathrm{~d}, 1 \mathrm{H}, J=4 \mathrm{~Hz}, 5-\mathrm{H}), 4.71(\mathrm{~d}, 1 \mathrm{H}, J=4 \mathrm{~Hz}, 4-\mathrm{H}), 4.18$ (q, $\left.2 \mathrm{H}, \mathrm{OCH}_{2}\right), 4.10\left(\mathrm{q}, 2 \mathrm{H}, \mathrm{OCH}_{2}\right), 3.76\left(\mathrm{~s}, 3 \mathrm{H}, \mathrm{OCH}_{3}\right), 1.24\left(\mathrm{t}, 3 \mathrm{H}, \mathrm{CH}_{3}\right), 1.07(\mathrm{t}, 3 \mathrm{H}$, 
$\left.\mathrm{CH}_{3}\right) ; \delta_{\mathrm{C}} 170.5,168.7(\mathrm{C}=\mathrm{O}), 161.4(\mathrm{C}-3), 161.2,126.2(\mathrm{ArC}), 129.6,113.8(4 \mathrm{ArCH})$, 62.3, $62.1\left(\mathrm{OCH}_{2}\right), 59.4(\mathrm{C}-5), 55.3\left(\mathrm{OCH}_{3}\right), 53.5(\mathrm{C}-4), 14.0,13.9\left(\mathrm{CH}_{3}\right) ; \mathrm{m} / \mathrm{z} 337$ $\left(M^{+}\right), 218,165\left(\mathrm{ArCNS}^{+}\right), 133\left(\mathrm{ArCN}^{+}\right)$.

Diethyl (E)-3-(4-methylphenyl)-2-isothiazoline-4,5-dicarboxylate (3c). Oil (Found: $M^{+}$, 321.1034. $\mathrm{C}_{16} \mathrm{H}_{19} \mathrm{NO}_{4} \mathrm{~S}$ requires $\left.\mathrm{M} 321.10347\right) ; v_{\max }(\mathrm{Nujol}) 1735 \mathrm{~cm}^{-1}(\mathrm{C}=\mathrm{O}) ; \delta_{\mathrm{H}}$ 7.7 (d, 2H, ArH), 7.1 (d, 2H, ArH), 5.1 (d, 1H, J=4 Hz, 5-H), 4.8 (d, 1H, J=4 Hz, 4$\mathrm{H}), 4.2\left(\mathrm{q}, 4 \mathrm{H}, \mathrm{OCH}_{2}\right), 2.3\left(\mathrm{~s}, 3 \mathrm{H}, \mathrm{CH}_{3}\right), 1.2\left(\mathrm{t}, 6 \mathrm{H}, \mathrm{CH}_{3}\right) ; \delta_{\mathrm{C}} 170.0,168.2(\mathrm{C}=\mathrm{O}), 161.5$ (C-3), 140.0, 130.2 (ArC), 128.7, 127.5 (4 ArCH), 61.8, $61.6\left(\mathrm{OCH}_{2}\right), 59.1$ (C-5), 53.2 (C-4), $20.9\left(\mathrm{CH}_{3}\right), 13.4\left(2 \mathrm{CH}_{3}\right) ; \mathrm{m} / \mathrm{z} 321\left(\mathrm{M}^{+}\right)$.

Diethyl (E)-3-(4-chlorophenyl)-2-isothiazoline-4,5-dicarboxylate (3d). Mp $49-50{ }^{\circ} \mathrm{C}$ (from EtOH) (Found: C, 52.9; H, 4.7; N, 4.0. $\mathrm{C}_{15} \mathrm{H}_{16} \mathrm{ClNO}_{4} \mathrm{~S}$ requires $\mathrm{C}, 52.7 ; \mathrm{H}, 4.7$; $\mathrm{N}, 4.1 \%) ; v_{\max }(\mathrm{Nujol}) 1733 \mathrm{~cm}^{-1}(\mathrm{C}=\mathrm{O}) ; \delta_{\mathrm{H}} 7.68(\mathrm{~d}, 2 \mathrm{H}, J=8.5 \mathrm{~Hz}, \mathrm{ArH}), 7.30(\mathrm{~d}, 2 \mathrm{H}$, $J=8.5 \mathrm{~Hz}, \mathrm{ArH}), 5.10(\mathrm{~d}, 1 \mathrm{H}, J=4 \mathrm{~Hz}, 5-\mathrm{H}), 4.76$ (d, 1H, $J=4 \mathrm{~Hz}, 4-\mathrm{H}), 4.22$ (q, 2H, $\left.\mathrm{OCH}_{2}\right), 4.10\left(\mathrm{q}, 2 \mathrm{H}, \mathrm{OCH}_{2}\right), 1.25\left(\mathrm{t}, 3 \mathrm{H}, \mathrm{CH}_{3}\right), 1.08\left(\mathrm{t}, 3 \mathrm{H}, \mathrm{CH}_{3}\right) ; \delta_{\mathrm{C}} 170.3,168.3$ $(\mathrm{C}=\mathrm{O}), 160.6$ (C-3), 136.2, $131.5(\mathrm{ArC}), 129.2,128.7$ (4 ArCH), 62.4, $62.3\left(\mathrm{OCH}_{2}\right)$, 59.1 (C-5), $53.6(\mathrm{C}-4), 14.0,13.9\left(\mathrm{CH}_{3}\right) ; \mathrm{m} / \mathrm{z} 343$ \& $341\left(\mathrm{M}^{+}\right), 224$ \& 222, 196 \& 198 $\left(\mathrm{ArCNS}^{+}\right), 171 \& 169\left(\mathrm{ArCN}^{+}\right)$.

Diethyl (E)-3-methyl-2-isothiazoline-4,5-dicarboxylate (3e). Bp $95{ }^{\circ} \mathrm{C}$ at 0.015 mmHg (Found: $\mathrm{C}, 49.1 ; \mathrm{H}, 6.2 ; \mathrm{N}, 5.8 . \mathrm{C}_{10} \mathrm{H}_{15} \mathrm{NO}_{4} \mathrm{~S}$ requires $\mathrm{C}, 49.0 ; \mathrm{H}, 6.2 ; \mathrm{N}, 5.7 \%$ ); $v_{\max }\left(\right.$ Nujol) $1730 \mathrm{~cm}^{-1}(\mathrm{C}=\mathrm{O}) ; \delta_{\mathrm{H}} 4.83(\mathrm{~d}, 1 \mathrm{H}, J=6 \mathrm{~Hz}, 5-\mathrm{H}), 4.53(\mathrm{~d}, 1 \mathrm{H}, J=6 \mathrm{~Hz}, 4-$ $\mathrm{H}), 4.22\left(\mathrm{q}, 2 \mathrm{H}, \mathrm{OCH}_{2}\right), 4.17\left(\mathrm{q}, 2 \mathrm{H}, \mathrm{OCH}_{2}\right), 2.15\left(\mathrm{~s}, 3 \mathrm{H}, \mathrm{CH}_{3}\right), 1.80\left(\mathrm{t}, 3 \mathrm{H}, \mathrm{CH}_{3}\right), 1.40$ $\left(\mathrm{t}, 3 \mathrm{H}, \mathrm{CH}_{3}\right) ; \delta_{\mathrm{C}} 170.6,167.9(\mathrm{C}=\mathrm{O}), 162.3(\mathrm{C}-3), 63.4(\mathrm{C}-5), 62.2\left(2 \mathrm{OCH}_{2}\right), 52.0(\mathrm{C}-$ 4), $20.0\left(\mathrm{CH}_{3}\right), 14.1\left(2 \mathrm{CH}_{3}\right) ; \mathrm{m} / \mathrm{z} 245\left(M^{+}\right), 126,73\left(\mathrm{MeCNS}^{+}\right)$.

Diethyl (E)-3-propyl-2-isothiazoline-4,5-dicarboxylate (3f). Bp $107{ }^{\circ} \mathrm{C}$ at 0.015 mmHg (Found: C, 52.8; H, 7.0; N, 5.1. $\mathrm{C}_{12} \mathrm{H}_{19} \mathrm{NO}_{4} \mathrm{~S}$ requires $\mathrm{C}, 52.7 ; \mathrm{H}, 7.0 ; \mathrm{N}, 5.1 \%$ ); $v_{\max }\left(\right.$ Nujol) $1735 \mathrm{~cm}^{-1}(\mathrm{C}=\mathrm{O}) ; \delta_{\mathrm{H}} 4.79(\mathrm{~d}, 1 \mathrm{H}, J=5 \mathrm{~Hz}, 5-\mathrm{H}), 4.50(\mathrm{~d}, 1 \mathrm{H}, J=5 \mathrm{~Hz}, 4-$ $\mathrm{H}), 4.21$ (q, 2H, $\mathrm{OCH}_{2}$ ), 4.16 (q, 2H, $\left.\mathrm{OCH}_{2}\right), 2.45$ (t, 2H, $\left.\mathrm{CH}_{2} \mathrm{CH}_{2} \mathrm{CH}_{3}\right), 1.90-1.48$ (m, $\left.2 \mathrm{H}, \mathrm{CH}_{2} \mathrm{CH}_{2} \mathrm{CH}_{3}\right), 1.28\left(\mathrm{t}, 3 \mathrm{H}, \mathrm{CH}_{3}\right), 1.24\left(\mathrm{t}, 3 \mathrm{H}, \mathrm{CH}_{3}\right), 0.92\left(\mathrm{t}, 3 \mathrm{H}, \mathrm{CH}_{3}\right) ; \delta_{\mathrm{C}} 170.7$, $168.0(\mathrm{C}=\mathrm{O}), 166.1(\mathrm{C}-3), 62.3(\mathrm{C}-5), 62.2\left(2 \mathrm{OCH}_{2}\right), 51.9(\mathrm{C}-4), 35.6,19.8\left(\mathrm{CH}_{2}\right), 14.1$ $\left(\mathrm{CH}_{3}\right), 13.7\left(2 \mathrm{CH}_{3}\right) ; \mathrm{m} / \mathrm{z} 273\left(M^{+}\right)$.

Diethyl (E)-3-heptyl-2-isothiazoline-4,5-dicarboxylate (3g). Bp $170{ }^{\circ} \mathrm{C}$ at 0.05 mmHg (Found: $\mathrm{C}, 58.2 ; \mathrm{H}, 8.0 ; \mathrm{N}, 4.3 . \mathrm{C}_{16} \mathrm{H}_{27} \mathrm{NO}_{4} \mathrm{~S}$ requires $\mathrm{C}, 58.3 ; \mathrm{H}, 8.3 ; \mathrm{N}, 4.3 \%$ ); $v_{\max }\left(\right.$ Nujol) $1735 \mathrm{~cm}^{-1}(\mathrm{C}=\mathrm{O}) ; \delta_{\mathrm{H}} 4.80(\mathrm{~d}, 1 \mathrm{H}, J=5 \mathrm{~Hz}, 5-\mathrm{H}), 4.55(\mathrm{~d}, 1 \mathrm{H}, J=5 \mathrm{~Hz}, 4-$ $\mathrm{H}), 4.22\left(\mathrm{q}, 2 \mathrm{H}, \mathrm{OCH}_{2}\right), 4.17\left(\mathrm{q}, 2 \mathrm{H}, \mathrm{OCH}_{2}\right), 2.48\left(\mathrm{t}, 2 \mathrm{H}, \mathrm{CH}_{2}\left(\mathrm{CH}_{2}\right)_{5} \mathrm{CH}_{3}\right), 1.88-1.26$ $\left(\mathrm{m}, 16 \mathrm{H}, \mathrm{CH}_{2}\left(\mathrm{CH}_{2}\right)_{5} \mathrm{CH}_{3} \& 2 \mathrm{CH}_{3}\right), 0.86\left(\mathrm{t}, 3 \mathrm{H}, \mathrm{CH}_{3}\right) ; \delta_{\mathrm{C}} 170.2,167.6(\mathrm{C}=\mathrm{O}), 165.8(\mathrm{C}-$ 
3), 61.9 (C-5), $61.7\left(2 \mathrm{OCH}_{2}\right), 51.5(\mathrm{C}-4), 33.3,31.3,28.7,28.6,26.0,22.2\left(\mathrm{CH}_{2}\right), 13.7$ $\left(3 \mathrm{CH}_{3}\right) ; \mathrm{m} / \mathrm{z} 329\left(M^{+}\right)$.

Dimethyl (E)-3-phenyl-2-isothiazoline-4,5-dicarboxylate (4a). Mp 59-60 ${ }^{\circ} \mathrm{C}$ (from EtOH) lit. ${ }^{5}$ a viscous oil of $\mathrm{mp} \sim 6^{\circ} \mathrm{C}$ ) (Found: $\mathrm{C}, 55.7 ; \mathrm{H}, 4.5 ; \mathrm{N}, 4.8 . \mathrm{C}_{13} \mathrm{H}_{13} \mathrm{NO}_{4} \mathrm{~S}$ requires C, 55.9; H, 4.7; N, 5.0\%); $v_{\max }(\mathrm{Nujol}) 1736 \mathrm{~cm}^{-1}(\mathrm{C}=\mathrm{O}) ; \mathrm{m} / \mathrm{z} 279\left(M^{\dagger}\right), 188$, $135\left(\mathrm{PhCNS}^{+}\right), 103\left(\mathrm{PhCN}^{+}\right)$.

Reaction of 4-methoxybenzonitrile sulfide (1b) with isobutyl acrylate. A solution of 5(4-methoxyphenyl)-1,3,4-oxathiazol-2-one (2b) $(4.0 \mathrm{~g}, 19 \mathrm{mmol})$ and isobutyl acrylate $(7.6 \mathrm{~g}, 59 \mathrm{mmol})$ in dry xylene $(100 \mathrm{ml})$ was heated under reflux in a nitrogen atmosphere until HPLC analysis showed that no oxathiazolone remained $(5 \mathrm{~h})$. The mixture was concentrated under vacuum to a yellow oil, which was suspended in cold ethanol to give white needles of 4-methoxybenzonitrile $(0.78,31 \%), \mathrm{mp} 59^{\circ} \mathrm{C}, v_{\max }$ (Nujol) $2210 \mathrm{~cm}^{-1}\left(\mathrm{C}^{\circ} \mathrm{N}\right), \mathrm{m} / \mathrm{z} 133\left(M^{+}\right)$. Chromatography of the residue (silica / heaxane- $\left.\mathrm{Et}_{2} \mathrm{O}, 1: 1\right)$ afforded the following compounds in order of elution.

Isobutyl 3-(4-methoxyphenyl)-2-isothiazoline-5-carboxylate (8b) $(1.17 \mathrm{~g}, 21 \%)$ as white crystals. Mp 51-52 ${ }^{\circ} \mathrm{C}$ (from ethanol) (Found: $\mathrm{C}, 61.5 ; \mathrm{H}, 6.5 ; \mathrm{N}, 4.7$. $\mathrm{C}_{15} \mathrm{H}_{19} \mathrm{NO}_{3} \mathrm{~S}$ requires $\left.\mathrm{C}, 61.4 ; \mathrm{H}, 6.5 ; \mathrm{N}, 4.8 \%\right) ; v_{\max }$ (Nujol) $1733 \mathrm{~cm}^{-1}(\mathrm{C}=\mathrm{O}) ; \delta_{\mathrm{H}} 7.66$ $(\mathrm{d}, 2 \mathrm{H}, J=9 \mathrm{~Hz}, \mathrm{ArH}), 6.85(\mathrm{~d}, 2 \mathrm{H}, J=9 \mathrm{~Hz}, \mathrm{ArH}), 4.54\left(\mathrm{dd}, 1 \mathrm{H}, J_{5,4 \mathrm{~b}}=11 \mathrm{~Hz}, J_{5,4 \mathrm{a}}=\right.$ $5 \mathrm{~Hz}, 5-\mathrm{H}), 3.99\left(\mathrm{dd}, 1 \mathrm{H}, J_{4 \mathrm{a}, 4 \mathrm{~b}}=17 \mathrm{~Hz}, 4 \mathrm{~b}-\mathrm{H}\right), 3.94\left(\mathrm{~d}, 2 \mathrm{H}, \mathrm{OCH}_{2}\right), 3.78(\mathrm{~s}, 3 \mathrm{H}$, $\left.\mathrm{OCH}_{3}\right), 3.46(\mathrm{dd}, 1 \mathrm{H}, 4 \mathrm{a}-\mathrm{H}), 1.95(\mathrm{~m}, 1 \mathrm{H}, \mathrm{CH}), 0.90\left(\mathrm{~d}, 6 \mathrm{H}, \mathrm{CH}_{3}\right) ; \delta_{\mathrm{C}} 171.5(\mathrm{C}=\mathrm{O})$, 161.6 (C-3), 160.9, 126.2 (ArC), 128.8, $113.7(4 \mathrm{ArCH}), 71.6\left(\mathrm{OCH}_{2}\right), 55.2\left(\mathrm{OCH}_{3}\right)$, 48.8 (C-5), $41.0(\mathrm{C}-4), 27.5(\mathrm{CH}), 18.8\left(2 \mathrm{CH}_{3}\right) ; \mathrm{m} / \mathrm{z} 293\left(M^{+}\right), 165\left(\mathrm{ArCNS}^{+}\right), 133$ $\left(\mathrm{ArCN}^{+}\right)$.

Isobutyl 3-(4-methoxyphenyl)-2-isothiazoline-4-carboxylate (7b) $(0.33 \mathrm{~g}, 6 \%)$ as white needles. Mp $42-43{ }^{\circ} \mathrm{C}$ (from hexane) (Found: $\mathrm{C}, 61.3 ; \mathrm{H}, 6.5 ; \mathrm{N}, 4.6 . \mathrm{C}_{15} \mathrm{H}_{19} \mathrm{NO}_{3} \mathrm{~S}$ requires $\mathrm{C}, 61.4 ; \mathrm{H}, 6.5 ; \mathrm{N}, 4.8 \%) ; v_{\max }(\mathrm{Nujol}) 1730 \mathrm{~cm}^{-1}(\mathrm{C}=\mathrm{O}) ; \delta_{\mathrm{H}} 7.73(\mathrm{~d}, 2 \mathrm{H}, J=9$ $\mathrm{Hz}, \operatorname{ArH}), 6.88(\mathrm{~d}, 2 \mathrm{H}, J=9 \mathrm{~Hz}, \mathrm{ArH}), 4.65\left(\mathrm{dd}, 1 \mathrm{H}, J_{4,5 \mathrm{a}}=9 \mathrm{~Hz}, J_{4,5 \mathrm{~b}}=7 \mathrm{~Hz}, 4-\mathrm{H}\right)$, 3.92-3.70 (m, 6H, 5a-H, 5b-H, $\left.\mathrm{OCH}_{2}\right), 3.80\left(\mathrm{~s}, 3 \mathrm{H}, \mathrm{OCH}_{3}\right), 1.82(\mathrm{~m}, 1 \mathrm{H}, \mathrm{CH}), 0.80(\mathrm{~d}$, $\left.6 \mathrm{H}, \mathrm{CH}_{3}\right) ; \delta_{\mathrm{C}} 169.8(\mathrm{C}=\mathrm{O}), 161.8(\mathrm{C}-3), 160.7,126.3(\mathrm{ArC}), 128.9,113.6(4 \mathrm{ArCH})$, $71.5\left(\mathrm{OCH}_{2}\right), 56.9(\mathrm{C}-4), 55.1\left(\mathrm{OCH}_{3}\right), 37.4(\mathrm{C}-5), 27.3(\mathrm{CH}), 18.6\left(2 \mathrm{CH}_{3}\right) ; \mathrm{m} / \mathrm{z} 293$ $\left(M^{+}\right), 165\left(\mathrm{ArCNS}^{+}\right), 133\left(\mathrm{ArCN}^{+}\right)$.

\section{Reaction of nitrile sulfides with diethyl maleate}

4-Methoxybenzonitrile sulfide (1b).- A suspension of 5-(4-methoxyphenyl)-1,3,4oxathiazol-2-one $(2 \mathrm{~b})(2.0 \mathrm{~g}, 9.6 \mathrm{mmol})$ and diethyl maleate $(16.5 \mathrm{~g}, 9.6 \mathrm{mmol})$ in dry xylene $(150 \mathrm{ml})$ was heated under reflux in a nitrogen atmosphere until HPLC analysis showed that no oxathiazolone remained $(5 \mathrm{~h})$. The mixture was concentrated under 
vacuum to a brown oil which was chromatographed on silica. Elution with $\mathrm{CH}_{2} \mathrm{Cl}_{2}$ yielded a yellow oil which solidified on treatment with cold ethanol to give a white crystalline solid, which was identified as diethyl (E)-3-(4-methoxyphenyl)-2isothiazoline-4,5-dicarboxylate (3b) $(23 \%)$ by comparison of its physical and spectroscopic properties with those of an authentic sample; mp and mixed mp $45-46{ }^{\circ} \mathrm{C}$. The reaction was repeated in the presence of an internal standard [dimethyl 3-(4chlorophenyl)isothiazole-4,5-dicarboxylate (9d)] to determine the yield of isothiazoline $3 \mathrm{~b}(32 \%)$ and 4-methoxybenzonitrile (67\%) by HPLC analysis. 4-Methylbenzonitrile sulfide (1c).- The corresponding reaction of 5-(4-methylphenyl)-1,3,4-oxathiazol-2-one (2c) and diethyl maleate afforded a brown oil which was shown by HLPC analysis to contain 4-methylbenzonitrile $(80 \%)$ together with traces $(<5 \%)$ of diethyl $(E)-3-(4-$ methylphenyl)-2-isothiazoline-4,5-dicarboxylate (3c). ${ }^{1} \mathrm{H} \quad$ NMR of recovered dipolarophile: $\delta_{\mathrm{H}} 6.0(\mathrm{~s}, 2 \mathrm{H}, \mathrm{CH}=), 3.8\left(\mathrm{q}, 4 \mathrm{H}, \mathrm{OCH}_{2}\right), 0.9\left(\mathrm{t}, 6 \mathrm{H}, \mathrm{CH}_{3}\right)$, indistinguishable from that of authentic diethyl fumarate. A sample of diethyl maleate $\left[\delta_{\mathrm{H}} 6.4(\mathrm{~s}, 2 \mathrm{H}, \mathrm{CH}=), 3.8\left(\mathrm{q}, 4 \mathrm{H}, \mathrm{OCH}_{2}\right), 0.9\left(\mathrm{t}, 6 \mathrm{H}, \mathrm{CH}_{3}\right)\right]$ was recovered unchanged after heating for $1 \mathrm{~h}$ under reflux $\left(\sim 225^{\circ} \mathrm{C}\right)$ in an atmosphere of nitrogen. A repeat experiment in the presence of sulfur afforded only diethyl fumarate.

Reaction of 4-methylbenzonitrile sulfide (1c) with (Z)-1,2-bis(phenylsulfonyl)ethene. A suspension of 5-(4-methylphenyl)-1,3,4-oxathiazol-2-one (2c) (685 mg, $3.5 \mathrm{mmol}$ ) and $(Z)-1,2$-bis(phenylsulfonyl)-ethene $(3.0 \mathrm{~g}, 9.7 \mathrm{mmol})$ in dry xylene $(40 \mathrm{ml})$ was heated under reflux in a nitrogen atmosphere for $20 \mathrm{~h}$. Removal of the solvent under vacuum gave a dark brown solid which was extracted with $\mathrm{CH}_{2} \mathrm{Cl}_{2}$. The solid residue $(1.42 \mathrm{~g})$ was identified as (E)-1,2-bis(phenylsulfonyl)ethene, mp $211^{\circ} \mathrm{C}$ (lit. ${ }^{13} 223{ }^{\circ} \mathrm{C}$ ); $\delta_{\mathrm{H}}$ 8.0-7.5 (m, 10H, PhH), $7.4(\mathrm{~s}, 2 \mathrm{H}, \mathrm{CH}=) ; \delta_{\mathrm{C}} 140.4(\mathrm{CH}=), 139.6(\mathrm{PhC}), 134.8$, 129.7, $128.4(5 \mathrm{PhCH})$. Chromatography of the filtrate (silica / hexane- $\mathrm{CH}_{2} \mathrm{Cl}_{2}$ 1:1) afforded in order of elution: sulfur $(0.09 \mathrm{~g})$, 4-methylbenzonitrile $(0.59 \mathrm{~g}),(E)-1,2-$ bis(phenylsulfonyl)ethene $\quad(0.25 \quad \mathrm{~g}), \quad$ and $\quad 4$-(phenylsulfonyl)-3-(4methylphenyl)isothiazole (5) (0.42 g, 38\%) (Found: $M^{+}, 315.0383 . \mathrm{C}_{16} \mathrm{H}_{13} \mathrm{NO}_{2} \mathrm{~S}_{2}$ requires M 315.03877); $v_{\max }(\mathrm{Nujol}) 1340,1120 \mathrm{~cm}^{-1}\left(\mathrm{SO}_{2}\right) ; \delta_{\mathrm{H}} 9.45(\mathrm{~s}, 1 \mathrm{H}, 5-\mathrm{H}), 8.4-$ 7.4 (m, 9H, ArH), 2.3 (s, 3H, $\mathrm{CH}_{3}$ ); $\delta_{\mathrm{C}} 156.2$ (C-3), 139.1 (C-4), 139.9, 130.3 (ArC), 132.7, 128.9, 128.3, 128.0, 127.3, $127.9(9 \mathrm{ArCH}), 20.6\left(\mathrm{CH}_{3}\right) ; \mathrm{m} / \mathrm{z} 315\left(M^{+}\right)$. A sample of (Z)-1,2-bis(phenylsulfonyl)ethene was recovered unchanged after heating for $20 \mathrm{~h}$ in xylene under reflux in an atmosphere of nitrogen. 
Thermolysis of 5-(4-methylphenyl)-1,3,4-oxathiazol-2-one (2c) with (E) and (Z)stilbenes.

(E)-stilbene.- A suspension of oxathiazolone $2 \mathrm{c}(750 \mathrm{mg}, 3.9 \mathrm{mmol})$ and $(E)$-stilbene $(1.425 \mathrm{~g}, 7.9 \mathrm{mmol})$ in xylene $(35 \mathrm{ml})$ was heated under reflux for $17.5 \mathrm{~h}$. From the reaction mixture were isolated by crystallisation and chromatography: $(E)$-stilbene $\left(90 \%\right.$, mp and mixed mp $121-122{ }^{\circ} \mathrm{C}$, lit. $\left.{ }^{14} 122-124{ }^{\circ} \mathrm{C}\right)$, sulfur $(95 \%)$ and $4-$ methylbenzonitrile (92\%). (Z)-stilbene.- A solution of oxathiazolone $2 \mathrm{c}(1.44 \mathrm{~g}, 7.5$ $\mathrm{mmol})$ and $(Z)$-stilbene $(2 \mathrm{ml}, 11.2 \mathrm{mmol})$ in xylene $(40 \mathrm{ml})$ was heated under reflux for $7 \mathrm{~h}$. From the reaction mixture were isolated by crystallisation and chromatography: (E)-stilbene (95\%, $\mathrm{mp}$ and mixed $\mathrm{mp} 121-122{ }^{\circ} \mathrm{C}$ ), sulfur (95\%) and 4methylbenzonitrile (90\%); residual (Z)-stilbene was not detected.

General procedure for Oxidation of 2-isothiazolines to isothiazoles using aq. NaOCl. To a solution of the oxathiazolone $(1.43 \mathrm{mmol})$ dissolved in $\mathrm{CH}_{2} \mathrm{Cl}_{2}(30 \mathrm{ml})$ was added $8 \%$ aq. $\mathrm{NaOCl}(30 \mathrm{ml})$. The mixture was stirred vigorously until no isothiazoline was detected by TLC or HPLC. The organic layer was separated, washed (water), dried $\left(\mathrm{MgSO}_{4}\right)$, and concentrated to an oil which was chromatographed on silica. Elution with $\mathrm{CH}_{2} \mathrm{Cl}_{2}$ yielded the isothiazole which was crystallised form cold ethanol. The reaction conditions and product yields are given in Table 2.

Dimethyl 3-phenylisothiazole-4,5-dicarboxylate (9a). Mp and mixed mp $72-73{ }^{\circ} \mathrm{C}$ (lit. $^{2 \mathrm{a}}$ $\left.72-73{ }^{\circ} \mathrm{C}\right)$.

Diethyl 3-(4-methoxyphenyl)isothiazole-4,5-dicarboxylate (10b). $\mathrm{Mp} 42-43{ }^{\circ} \mathrm{C}$ (from EtOH) (Found: $\mathrm{C}, 57.1 ; \mathrm{H}, 5.2 ; \mathrm{N}, 4.2 . \mathrm{C}_{16} \mathrm{H}_{17} \mathrm{NO}_{5} \mathrm{~S}$ requires $\mathrm{C}, 57.3 ; \mathrm{H}, 5.1 ; \mathrm{N}, 4.2 \%$ ); $v_{\max }$ (Nujol) $1723 \mathrm{~cm}^{-1}(\mathrm{C}=\mathrm{O}) ; \delta_{\mathrm{H}} 7.65(\mathrm{~d}, 2 \mathrm{H}, J=9 \mathrm{~Hz}, \mathrm{ArH}), 6.92(\mathrm{~d}, 2 \mathrm{H}, J=9 \mathrm{~Hz}$, $\mathrm{ArH}), 4.37\left(\mathrm{~m}, 4 \mathrm{H}, \mathrm{OCH}_{2}\right), 3.80\left(\mathrm{~s}, 3 \mathrm{H}, \mathrm{OCH}_{3}\right), 1.36\left(\mathrm{t}, 3 \mathrm{H}, \mathrm{CH}_{3}\right), 1.30\left(\mathrm{t}, 3 \mathrm{H}, \mathrm{CH}_{3}\right)$; $\mathrm{m} / \mathrm{z} 335\left(M^{+}\right), 133\left(\mathrm{ArCN}^{+}\right)$.

General procedure for Oxidation of 2-isothiazolines to isothiazoles using aq $\mathrm{NaOCl}$ and a phase-transfer catalyst (benzyltriethylammonium chloride). To a solution of the oxathiazolone $(1.4 \mathrm{mmol})$ dissolved in $\mathrm{CH}_{2} \mathrm{Cl}_{2}(20 \mathrm{ml})$ was added $8 \%$ aq. $\mathrm{NaOCl}$ $(40 \mathrm{ml})$ and benzyltriethylammonium chloride $(0.2 \mathrm{mmol})$. The mixture was stirred vigorously until no isothiazoline was detected by HPLC analysis ( 3 weeks). The organic layer was separated, washed (water), dried $\left(\mathrm{MgSO}_{4}\right)$, and concentrated under vacuum. Solid products were purified by recrystallisation, and liquids by distillation under reduced pressure. The reaction conditions and product yields are given in Table B. 
Diethyl 3-phenylisothiazole-4,5-dicarboxylate (10a). Bp $175{ }^{\circ} \mathrm{C}$ at $0.04 \mathrm{mmHg}$ (Found: $\mathrm{C}, 58.8 ; \mathrm{H}, 4.9 ; \mathrm{N}, 4.8 . \mathrm{C}_{15} \mathrm{H}_{15} \mathrm{NO}_{4} \mathrm{~S}$ requires $\mathrm{C}, 59.0 ; \mathrm{H}, 5.0 ; \mathrm{N}, 4.6 \%$ ); $v_{\max }$ (Nujol) $1735 \mathrm{~cm}^{-1}(\mathrm{C}=\mathrm{O})$; $\delta_{\mathrm{H}} 7.80-7.60(\mathrm{~m}, 2 \mathrm{H}, \mathrm{PhH}), 7.50-7.36(\mathrm{~m}, 3 \mathrm{H}, \mathrm{PhH}), 4.39$ (q, $\left.2 \mathrm{H}, \mathrm{OCH}_{2}\right), 4.37\left(\mathrm{q}, 2 \mathrm{H}, \mathrm{OCH}_{2}\right), 1.37\left(\mathrm{t}, 3 \mathrm{H}, \mathrm{CH}_{3}\right), 1.30\left(\mathrm{t}, 3 \mathrm{H}, \mathrm{CH}_{3}\right) ; \mathrm{m} / \mathrm{z} 335\left(M^{+}\right)$, $133\left(\mathrm{ArCN}^{+}\right)$.

Diethyl 3-(4-chlorophenyl)isothiazole-4,5-dicarboxylate (10d). Mp 46-47 ${ }^{\circ} \mathrm{C}$ (from EtOH) (Found: $\mathrm{C}, 52.7 ; \mathrm{H}, 4.1 ; \mathrm{N}, 4.0 . \mathrm{C}_{15} \mathrm{H}_{14} \mathrm{ClNO}_{4} \mathrm{~S}$ requires $\mathrm{C}, 53.0 ; \mathrm{H}, 4.2 ; \mathrm{N}$, $4.1 \%) ; v_{\max }(\mathrm{Nujol}) 1730 \mathrm{~cm}^{-1}(\mathrm{C}=\mathrm{O}) ; \delta_{\mathrm{H}} 7.64(\mathrm{~d}, 2 \mathrm{H}, J=8.5 \mathrm{~Hz}, \mathrm{ArH}), 7.38(\mathrm{~d}, 2 \mathrm{H}, J=$ $8.5 \mathrm{~Hz}, \mathrm{ArH}), 4.39$ (q, 2H, $\left.\mathrm{OCH}_{2}\right), 4.37$ (q, 2H, $\left.\mathrm{OCH}_{2}\right), 1.36\left(\mathrm{t}, 3 \mathrm{H}, \mathrm{CH}_{3}\right), 1.29(\mathrm{t}, 3 \mathrm{H}$, $\left.\mathrm{CH}_{3}\right) ; \mathrm{m} / \mathrm{z} 341 \& 339\left(M^{+}\right)$.

Diethyl 3-methyl-2-isothiazole-4,5-dicarboxylate (10e). Bp $80{ }^{\circ} \mathrm{C}$ at $0.01 \mathrm{mmHg}$ (Found: C, 49.3; H, 5.4; N, 5.6. $\mathrm{C}_{10} \mathrm{H}_{13} \mathrm{NO}_{4} \mathrm{~S}$ requires $\mathrm{C}, 49.4 ; \mathrm{H}, 5.4 ; \mathrm{N}, 5.8 \%$ ); $v_{\max }$ (Nujol) $1725 \mathrm{~cm}^{-1}(\mathrm{C}=\mathrm{O}) ; \delta_{\mathrm{H}} 4.37\left(\mathrm{q}, 2 \mathrm{H}, \mathrm{OCH}_{2}\right), 4.35\left(\mathrm{q}, 2 \mathrm{H}, \mathrm{OCH}_{2}\right), 2.52(\mathrm{~s}, 3 \mathrm{H}$, $\left.\mathrm{CH}_{3}\right), 1.34$ (t, 6H, $\left.\mathrm{CH}_{3}\right) ; \mathrm{m} / \mathrm{z} 243\left(M^{+}\right)$.

Diethyl 3-propyl-2-isothiazole-4,5-dicarboxylate (10f). $\mathrm{Bp} 120{ }^{\circ} \mathrm{C}$ at $0.04 \mathrm{mmHg}$ (Found: $\mathrm{C}, 53.2 ; \mathrm{H}, 6.3 ; \mathrm{N}, 5.4 . \mathrm{C}_{12} \mathrm{H}_{17} \mathrm{NO}_{4} \mathrm{~S}$ requires $\mathrm{C}, 53.1 ; \mathrm{H}, 6.3 ; \mathrm{N}, 5.2 \%$ ); $v_{\max }$ (Nujol) $1730 \mathrm{~cm}^{-1}(\mathrm{C}=\mathrm{O})$; $\delta_{\mathrm{H}} 4.42\left(\mathrm{q}, 2 \mathrm{H}, \mathrm{OCH}_{2}\right), 4.38\left(\mathrm{q}, 2 \mathrm{H}, \mathrm{OCH}_{2}\right), 2.87(\mathrm{t}, 2 \mathrm{H}$, $\left.\mathrm{CH}_{2} \mathrm{CH}_{2} \mathrm{CH}_{3}\right), 1.94-1.56\left(\mathrm{~m}, 2 \mathrm{H}, \mathrm{CH}_{2} \mathrm{CH}_{2} \mathrm{CH}_{3}\right), 1.38\left(\mathrm{t}, 6 \mathrm{H}, \mathrm{CH}_{3}\right), 0.98\left(\mathrm{t}, 3 \mathrm{H}, \mathrm{CH}_{3}\right)$; $\mathrm{m} / \mathrm{z} 271\left(M^{+}\right)$.

Diethyl 3-heptyl-2-isothiazole-4,5-dicarboxylate $(10 \mathrm{~g}) . \mathrm{Bp} 160{ }^{\circ} \mathrm{C}$ at $0.04 \mathrm{mmHg}$ (Found: $\mathrm{C}, 58.7 ; \mathrm{H}, 7.7 ; \mathrm{N}, 4.3 . \mathrm{C}_{16} \mathrm{H}_{25} \mathrm{NO}_{4} \mathrm{~S}$ requires $\mathrm{C}, 58.5 ; \mathrm{H}, 7.7 ; \mathrm{N}, 4.5 \%$ ); $v_{\max }$ (Nujol) $1725 \mathrm{~cm}^{-1}(\mathrm{C}=\mathrm{O}) ; \delta_{\mathrm{H}} 4.42\left(\mathrm{q}, 2 \mathrm{H}, \mathrm{OCH}_{2}\right), 4.38\left(\mathrm{q}, 2 \mathrm{H}, \mathrm{OCH}_{2}\right), 2.90(\mathrm{t}, 2 \mathrm{H}$, $\left.\mathrm{CH}_{2}\left(\mathrm{CH}_{2}\right)_{5} \mathrm{CH}_{3}\right), 1.90-1.18\left(\mathrm{~m}, 16 \mathrm{H}, \mathrm{CH}_{2}\left(\mathrm{CH}_{2}\right)_{5} \mathrm{CH}_{3} \& 2 \mathrm{CH}_{3}\right), 0.89\left(\mathrm{t}, 3 \mathrm{H}, \mathrm{CH}_{3}\right) ; \mathrm{m} / \mathrm{z}$ $327\left(M^{+}\right)$.

Isobutyl 3-(4-methoxyphenyl)isothiaxole-4-carboxylate (11b). $\mathrm{Bp} 160{ }^{\circ} \mathrm{C}$ at 0.01 mmHg (Found: $\mathrm{C}, 62.0 ; \mathrm{H}, 5.9 ; \mathrm{N}, 4.9 . \mathrm{C}_{15} \mathrm{H}_{17} \mathrm{NO}_{3} \mathrm{~S}$ requires $\mathrm{C}, 61.8 ; \mathrm{H}, 5.9 ; \mathrm{N}, 4.8 \%$ ); $v_{\max }(\mathrm{Nujol}) 3105(\mathrm{C}-\mathrm{H}), 1727 \mathrm{~cm}^{-1}(\mathrm{C}=\mathrm{O}) ; \delta_{\mathrm{H}} 9.30(5-\mathrm{H}), 7.60(\mathrm{~d}, 2 \mathrm{H}, J=9 \mathrm{~Hz}, \mathrm{ArH})$, $6.90(\mathrm{~d}, 2 \mathrm{H}, J=9 \mathrm{~Hz}, \mathrm{ArH}), 4.02\left(\mathrm{~d}, 2 \mathrm{H}, \mathrm{OCH}_{2}\right), 3.82\left(\mathrm{~s}, 3 \mathrm{H}, \mathrm{OCH}_{3}\right), 1.91(\mathrm{~m}, 1 \mathrm{H}$, $\mathrm{CH}), 0.89\left(\mathrm{~d}, 6 \mathrm{H}, \mathrm{CH}_{3}\right) ; \delta_{\mathrm{C}} 168.0(\mathrm{C}=\mathrm{O}), 162.0(\mathrm{C}-3), 160.1,127.3(\mathrm{ArC}), 1555.5(\mathrm{C}-5)$, 130.2, 113.0 (4 ArCH), $70.9(\mathrm{OCH}), 55.0\left(\mathrm{OCH}_{3}\right), 27.4(\mathrm{CH}), 18.8\left(2 \mathrm{CH}_{3}\right) ; \mathrm{m} / \mathrm{z} 291$ $\left(M^{+}\right), 133\left(\mathrm{ArCN}^{+}\right)$.

Isobutyl 3-(4-methoxyphenyl)isothiaxole-5-carboxylate (12b) $\mathrm{Mp} 82-83{ }^{\circ} \mathrm{C}$ (Found: $\mathrm{C}, 61.5 ; \mathrm{H}, 5.9 ; \mathrm{N}, 4.7 . \mathrm{C}_{15} \mathrm{H}_{17} \mathrm{NO}_{3} \mathrm{~S}$ requires $\mathrm{C}, 61.8 ; \mathrm{H}, 5.9 ; \mathrm{N}, 4.7 \%$ ); $v_{\max }$ (Nujol) $3310(\mathrm{C}-\mathrm{H}), 1723 \mathrm{~cm}^{-1}(\mathrm{C}=\mathrm{O}) ; \delta_{\mathrm{H}} 7.92(4-\mathrm{H}), 7,82(\mathrm{~d}, 2 \mathrm{H}, J=9 \mathrm{~Hz}, \mathrm{ArH}), 6.84(\mathrm{~d}, 2 \mathrm{H}$, $J=9 \mathrm{~Hz}, \mathrm{ArH}), 4.05\left(\mathrm{~d}, 2 \mathrm{H}, \mathrm{OCH}_{2}\right), 3.89\left(\mathrm{~s}, 3 \mathrm{H}, \mathrm{OCH}_{3}\right), 2.10(\mathrm{~m}, 1 \mathrm{H}, \mathrm{CH}), 1.02$ (d, $\left.6 \mathrm{H}, \mathrm{CH}_{3}\right) ; \delta_{\mathrm{C}} 167.7(\mathrm{C}=\mathrm{O}), 160.8(\mathrm{C}-3), 157.4(\mathrm{C}-5), 160.1,127.1(\mathrm{ArC}), 128.2,114.2$ 
(4 ArCH), $71.7\left(\mathrm{OCH}_{2}\right), 55.2\left(\mathrm{OCH}_{3}\right), 27.7(\mathrm{CH}), 18.9\left(2 \mathrm{CH}_{3}\right) ; \mathrm{m} / \mathrm{z} 291\left(M^{+}\right), 133$ $\left(\mathrm{ArCN}^{+}\right)$.

\section{Acknowledgements}

We are grateful to the EPSRC for research and maintenance (M.C.McK \& J.F.R) grants.

\section{References}

1. For reviews of isothiazoles see: (a) Pain, D. L.; Peart, B. J.; Wooldridge, K. R. H. Comprehensive Heterocyclic Chemistry; 1984; vol. 6, chapter 4.17. (b) Chapman, R. F.; Peart, B. j. Comprehensive Heterocyclic Chemistry II; 1996; vol. 3, chapter 3.07.

2. Howe, R. K.; Gruner, T. A.; Carter, L. G.; Black, L. L.; Franz, J. E. J. Org. Chem. 1978, 43, 3736. (b) Yoshida, H.; Taketani, H.; Ogata, T.; Inokawa, S. Bull. Chem. Soc. Jpn. 1976, 49, 3124. (c) Sanders, M. J.; Dye, S. L.; Miller, A. G.; Grunwell, J. R. J. Org. Chem. 1979, 44, 510. (d) McKie, M. C.; Paton, R. M. J. Chem. Res. 1987, (S) 245, (M), 2051. (e) Brownsort, P. A.; Paton, R. M. J. Chem. Soc., Perkin Trans. 1, 1987, 2339. (f) Brownsort, P. A.; Paton, R. M.; Sutherland, A. G. J. Chem. Soc., Perkin Trans. 1, 1989, 1679 and references therein.

3. Damas, A. M.; Gould, R. O.; Harding, M. M.; Paton, R. M.; Ross, J. F.; Crosby, J. J. Chem. Soc., Perkin Trans. 1, 1981, 2991 and references therein.

4. For a review of nitrile sulfide chemistry see Paton, R. M. Chem. Soc. Rev. 1989, 18, 33.

5. Howe, R. H.; Franz, J. E. J. Org. Chem. 1978, 43, 3742.

6. Rahman, Clapp, L. J. Org. Chem. 1976, 41, 122.

7. Gould, R. O.; Paton, R. M.; Ross, J. F. unpublished observations.

8. Caramella, P.; Grünanger, P. In 1,3-Dipolar Cycloaddition Chemistry, ed.; Padwa, A. Wiley: New York, 1984 chapter 3.

9. Doyle, M. P.; Low, K. L.; Nishioka, L. I.; McVickar, M. B.; Liu, M. T. H. Tetrahedron Lett. 1986, 27, 4395.

10. Wentrup, C.; Kambouris, P. Chem. Rev. 1991, 91, 363.

11. Greig, D. J.; McPherson, M.; Paton, R. M.; Crosby, J. J. Chem. Soc., Perkin Trans. 1, 1985, 1205. 
12. Blake, A. J.; Boyd, E. C.; Gould, R. O.; Paton, R. M. J. Chem. Soc., Perkin Trans. 1, 1994, 2841.

13. De Lucchi, O.; Lucchini, V.; Pasquato, V.; Modena, G. J. Org. Chem. 1984, 49, 596.

14. Dictionary of Organic Compounds; Ed.; Buckingham, J. Chapman \& Hall: 1982. 